\title{
El poblamiento prehistórico del Campo de Gibraltar
}

\author{
M. Mas Cornellà ${ }^{1}$, S. Ripoll López ${ }^{2}$, G. Torra Colell ${ }^{3}$, \\ J.F. Jordá Pardo ${ }^{4}$, B. Gavilán Ceballos ${ }^{5}$ y J.C. Vera Rodriguez ${ }^{6}$
}

El poblamiento prehistórico en el Campo de Gibraltar ha sido conocido hasta hace relativamente poco tiempo por los trabajos pioneros de principios de siglo y algunas intervenciones arqueológicas esporádicas.

En este artículo presentamos una sintesis de los resultados obtenidos dentro del proyecto de investigación arqueológica Las manifestaciones rupestres prehistóricas de la zona gaditana (actividades arqueológicas autorizadas y subvencionadas por la Dirección General de Bienes Culturales de la Consejería de Cultura de la Junta de Andalucia), que viene desarrollándose en las sierras que bordean la antigua Laguna de la Janda desde 1986 (Mas Cornellà, 1986, 1987). Dentro de este proyecto interdisciplinar se documentan la totalidad de las estaciones con pinturas o grabados y su contexto arqueológico a partir de técnicas de investigación innovadoras, 10 que, junto a importantes y recientes descubrimientos, permite elaborar nuevas hipótesis en relación al poblamiento prehistórico.

El poblamiento prehistórico de las sierras del Campo de Gibraltar ha sido relativamente poco estudiado. Sólo podemos citar, refiriéndonos exclusivamente al área que nos ocupa, los trabajos pioneros de $\mathrm{H}$. Breuil,

\footnotetext{
1 Departamento de Prehistoria e Historia Antigua de la Universidad Nacional de Educación a Distancia.

2 Departamento de Prehistoria e Historia Antigua de la Universidad Nacional de Educación a Distancia.

3 Proyecto de investigación arqueológica Las manifestaciones rupestres prehistóricas de la zona gaditana.

4 Departamento de Prehistoria, Historia Antigua y Arqueología de la Universidad de Salamanca.

5 Departamento de Historia de la Antigüedad, del Arte, Geografía Física y Antropología de la Universidad de Huelva. Cordoba.

6 Departamento de Ciencias Humanas Experimentales y del Territorio de la Universidad de
} 
M.C. Burkitt, W. Verner, J. Cabré, E. Hernández-Pacheco y C. de Mergelina, que estudiaron los conocidos monumentos megalíticos esporádicos, algunas veces en relación a abrigos con pinturas rupestres (Hernández-Pacheco y Cabré, 1913; Cabré y Hernández-Pacheco, 1914; Breuil y Verner, 1917; Mergelina, 1924; Breuil y Burkitt, 1929). Destacan en este panorama la necrópolis de Los Algarbes (Posac Mon, 1975; Mata Almonte, 1991, 1995) y tres yacimientos al aire libre estudiados recientemente, las Cuevas de Levante, la Cubeta de la Paja y el Río Palmores (Mas Cornellà y Sanchidrián Torti, 1990; Ripoll López y otros, 1991a; Pérez Ramos, 1995; Ramos Muñoz y otros, 1995c).

En las sierras del Campo de Gibraltar, dentro del Parque Natural de los Alcornocales, existen algo más de ciento veinte estaciones con manifestaciones rupestres pintadas y/o grabadas (Mas Cornellà, inédito). La mayoría de ellas fueron dadas a conocer por J. Cabré, E. HernándezPacheco, H. Breuil, M.C. Burkitt y W. Verner (Molina, 1913; Verner 1914a, 1914b, 1914c; Cabré y Hernández-Pacheco, 1914; Breuil y Burkitt, 1929), otras han sido divulgadas más recientemente en estudios de desiguales características (Mas Cornellà y otros, 1995b).

No entraremos en consideraciones sobre aspectos tan importantes como la conservación de estos documentos, ya que nos hemos referido a esta problemática en anteriores artículos (Mas Cornellà, 1989a; Mas Cornellà y otros, 1994). No hace falta insistir en que todos estos yacimientos están especialmente desprotegidos y su estado de conservación es precario, debido principalmente a la acción antrópica, que en algunas ocasiones está causando graves deterioros irreversibles.

Es una opinión generalizada que las pinturas rupestres de las sierras que bordean la antigua Laguna de la Janda constituyen un caso aparte dentro del arte postpaleolítico de la Península lbérica (Acosta Martínez, $1968,1984)$. Junto a determinados motivos relacionados con el denominado fenómeno esquemático presentan unas fases con representaciones de tendencia naturalista (figuras humanas, cérvidos, caprinos y otros zoomorfos) que se diferencian de las consideradas esquemáticas y otras que no pueden compararse tipológicamente con las que se localizan aquí (Baidellou, 1989), con aspectos originales, muy poco frecuentes, como la representación de aves o la utilización de pintura blanca para plasmar abstracciones, como podemos ver en la Cueva del Tajo de las Figuras. Teniendo en cuenta la cada vez más evidente unidad conceptual entre el Neolítico y el Calcolítico (paralelos muebles y cerámicos) podemos establecer, a modo de hipótesis, un horizonte cronológico y cultural que comprende desde el Neolítico hasta la Edad del Bronce para estas manifesta- 


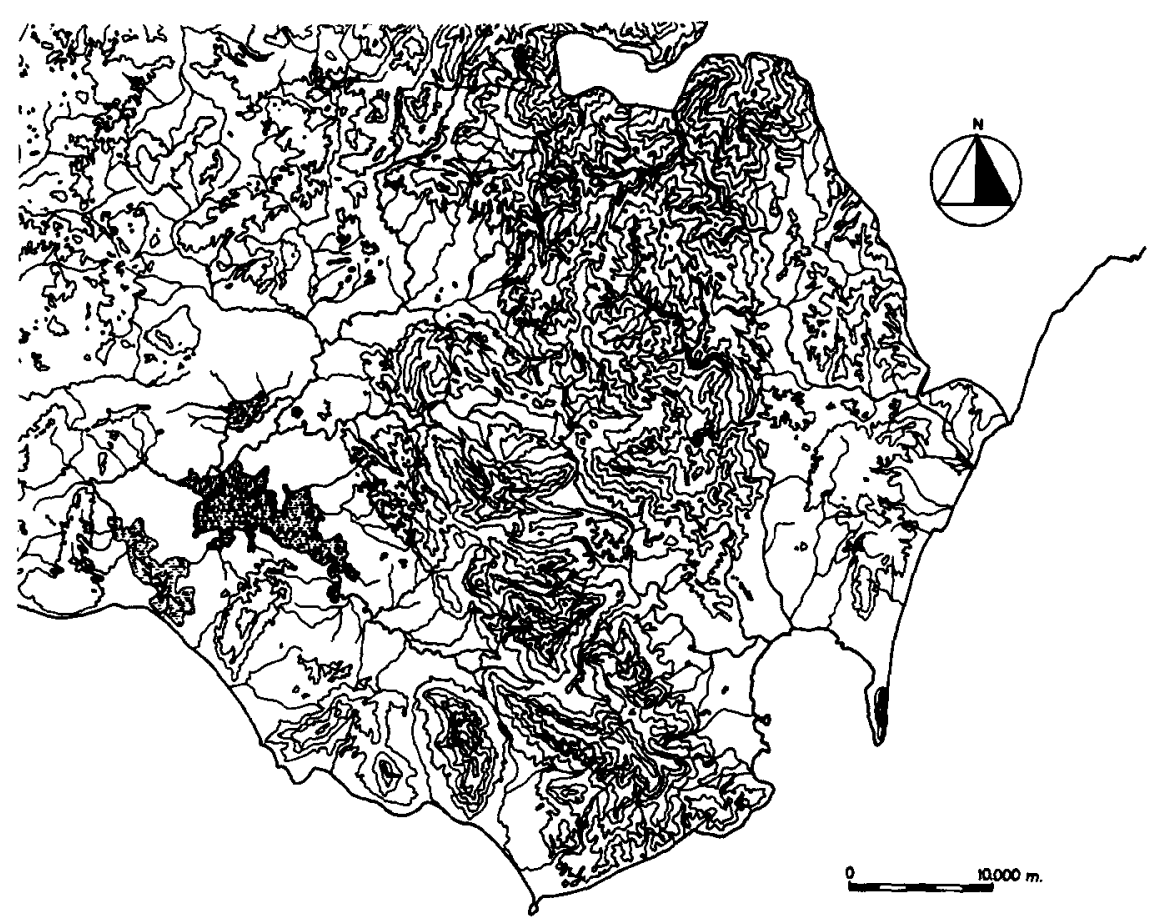

Figura 1. Sierras del Campo de Gibraltar y reconstrucción de la antigua Laguna de la Janda (Dibujo realizado a partir de la cartografía siguiente: Mapa Topográfico Nacional, Escala 1:50.000, Vejer de la Frontera, (1.073), Dirección General del Instituto Geográfico Nacional, Madrid (segunda edición), 1955; Mapa Topográfico Nacional, Escala 1:50.000,

Las Habanas, (1.074), Dirección General del Instituto Geográfico Nacional, Madrid (segunda edición), 1960; y Mapa provincial, Escala 1:200.000. Cádiz y Ciudad de Ceuta, Dirección General del instituto Geográfico Nacional, Madrid (tercera edición), 1988.

ciones. Recordemos que casi todos los investigadores aceptan una fecha en torno al 1000 a. de J.C. para las embarcaciones del Abrigo de la Laja Alta (Corzo Sánchez y Giles Pacheco, 1978; Barroso Ruiz, 1980; Almagro Gorbea, 1988; Ripoll Perelló, 1990; Mas Cornellà, 1993a).

Todos estos accidentes geográficos están situados en las Unidades Alóctonas del Campo de Gibraltar, dentro de las Cordilleras Béticas. Estas unidades forman un conjunto de mantos y escamas completamente desenraizados, constituidos principalmente por formaciones cretácicas y ter- 
ciarias, en las cuales las de "tipo Flysch» tienen una muy importante representación (Fontboté y Vera, 1983). Una de estas unidades, que compone el Complejo del Campo de Gibraltar, es la “Unidad del Aljibe», situada entre el Senoniense (Cretácico Superior) y el Burdigaliense (Mioceno Medio), y es en una de las formaciones que culminan esta unidad en donde se desarrollan las cavidades que componen los conjuntos rupestres que nos ocupan.

La Formación Areniscas del Aljibe (Gavala, 1916, 1929; Fontboté, 1983; ITGE, 1991) llega a alcanzar una potencia de 2.000-1.500 m., y está formada predominantemente por areniscas de aspecto masivo, que son exclusivamente silíceas y tienen una coloración blanquecina o amarillenta en fractura fresca, que pasa a parda por meteorización, con finas intercalaciones de materiales pelíticos de color marrón claro. Son unas areniscas constituidas por granos de cuarzo, bien redondeados, de tamaño pequeño a grueso, a veces conglomeráticas, con apenas matriz de limos y arenas de grano muy fino y mala cementación, de tipo ferruginoso, no muy consistentes, por lo que la roca tiene una cierta friabilidad. Apenas contienen fósiles, por lo que su datación paleontológica es prácticamente imposible. No obstante el techo de los materiales que descansan por debajo de las Areniscas del Aljibe tiene una edad Aquitaniense, mientras que las arcillas con intercalaciones de arenas que se sitúan a techo de las Areniscas del Aljibe, tienen una edad Aquitaniense Superior-Burdigaliense. Por tanto las Areniscas del Aljibe se depositan una vez iniciado el Aquitaniense, terminando su sedimentación en un momento no precisado, anterior al Aquitaniense Superior Burdigaliense. Estas areniscas se pueden correlacionar claramente con el llamado Manto Numídico al otro lado del Estrecho de Gibraltar (Fontboté, 1983).

Las Areniscas del Aljibe están claramente tectonizadas por la Orogenia Alpina, encontrándose en la zona de Sierra Momia formando un sinclinal tumbado, cuyo eje tiene una dirección NW-SE, presentando un buzamiento invertido de $55^{\circ}$. En Sierra del Niño se observa un plegamiento con sistemas de sinclinales y anticlinales tumbados como el de las Cuevas de Palomas. Esta formación se encuentra cortada por numerosas fallas normales de dirección EW y SW-NE, algunas de cierta extensión, produciendo desplazamientos verticales y horizontales en los materiales plegados. En los planos de estas fallas, claras zonas de debilidad, es frecuente el desarrollo de cavidades, como es el caso de la Cueva del Tajo de las Figuras. También se encuentra afectada esta formación por despegues que dan lugar a importantes cicatrices como la de la zona de Bacinete.

Geomorfológicamente las Areniscas del Aljibe destacan en el relieve, dando lugar a varias alineaciones montañosas como Sierra Momia y Sierra 
del Niño, limitadas por áreas deprimidas, cuyo mejor exponente es la Laguna de la Janda. Estas sierras presentan la peculiaridad de contener numerosas cavidades o abrigos rocosos, de pequeño tamaño, originadas por corrosión y erosión eólica, junto con superficies corroídas en extensión, dando lugar en conjunto a una morfología de tafonis en areniscas siliceas.

Partiendo de estas premisas en 1988 nos planteamos la realización de un proyecto de investigación aqueológica - Las manifestaciones rupestres prehistóricas de la zona gaditana (Mas Cornellà, 1986, 1986-1987, 1987, 1988a, 1988b, 1988-1989, 1989a, 1989b, 1990, 1991a, 1991b, 1993a, 1993b, en prensa; Mas Cornellà y Sanchidrián Torti, 1990; Mas Cornellà y Torra Colell, 1990, 1995; Ripoll López y otros, 1991a, 1991b; Jordá Pardo y otros, 1994; Mas Cornellà y otros, 1994, 1995a, 1995b, 1996; Sanchidrián Torti y Mas Cornellà, 1995; Mas Cornellà y Ripoll López, en prensa) - que nos permitiera aproximarnos al horizonte cronológico y cultural de las manifestaciones rupestres del área. Debido a la abundancia del material obtenido y procesado hemos tenido que limitar el proyecto a dos sierras, Sierra Momia y Sierra del Niño, y a algo más de treinta yacimientos, entre los que se encuentran los más importantes por la cantidad y características de los motivos que contienen - Conjunto rupestre del Tajo de las Figuras, Cuevas de los Ladrones o Pretinas, Abrigos de Bacinete, Cuevas de Palomas ...-, constituyendo una primera fase de nuestras investigaciones.

Desde un primer momento creímos que un estudio riguroso sobre arte prehistórico debe partir de una detallada y correcta documentación - hemos constatado muchos casos en los que una figura inadecuadamente reproducida, que en realidad no existe como tal, ha hecho correr ríos de tinta entre los estudiosos del arte prehistórico, encontrándonos, pués, cuando esto sucede, ante argumentos acertados y metodológicamente bien desarrollados que se basan en un dato falso-, precedida por un diagnóstico del estado de conservación de las figuras y las características del soporte rocoso de las pinturas y grabados, así como de los procesos que lo alteran. Estas investigaciones, que consideramos fundamentales al proceder a la reproducción y estudio directo de estos frágiles documentos, nos han revelado datos como la composición química y mineralógica de las rocas soporte, sus características intrínsecas y las causas de alteración, el impacto de la actividad fisiológica de las comunidades de criptógamas sobre los paneles con representaciones, o la incidencia de aves e insectos que construyen sus nidos en los abrigos (Mas Cornellà $y$ otros, 1994). 
Entre 1988 y 1991, durante sucesivas campañas de trabajo de campo, intervinimos en los yacimientos de Sierra Momia y Sierra del Niño. Esta documentación se basa en la situación cartográfica, la topografía de cada yacimiento y la elaboración de reproducciones de los paneles a partir de las fotografías impresionadas de cada uno de los motivos (Mas Cornellà, 1990). Durante las campañas de 1989 y 1990 se analizaron las reacciones de los pigmentos de las figuras, actuando con radiaciones de longitud de onda fuera del espectro visible, tanto en la banda infrarroja como en la ultravioleta (Mas Cornellà, 1989b, 1990). En 1992 y en función de la experiencia acumulada trabajamos nuevamente de forma puntual aplicando estas técnicas (Mas Cornellà, 1993b). Se consignan, en fichas especialmente diseñadas, abundantes apuntes sobre cada figura y anotaciones diversas de interés. Todo este material se procesa en el gabinete y se trata la información por ordenador, introduciéndola en bases de datos (Mas Cornellà, 1990).

La contextualización cultural de estas manifestaciones artísticas nos llevó a considerar el entorno arqueológico, aunque, de momento, éste no ha sido abordado de manera exahustiva. Presentamos aquí también un avance, que esperamos poder ampliar en futuras investigaciones y trabajos de campo, en relación al conocimiento del Paleolítico Superior de esta zona, del que sólo se poseían escasos, y en algunos casos conflictivos, datos del nivel B de Gorham's Cave (Gibraltar) (Waechter, 1953, 1964), de las pinturas de Cueva de Palomas 1 (Facinas, Tarifa) (Breuil y Burkitt, 1929; Breuil, 1974; Fortea Pérez, 1978; Santiago Vilchez, 1979-1980; Mas Cornellà y otros, 1995a; Sanchidrián Torti y Mas Cornellà, 1995) y de las de St Michel's Cave (Gibraltar) (Breuil, 1921, 1922).

En 1990 una prospección arqueológica superficial (Mas Cornellà y Sanchidrián Torti, 1990), realizada con la finalidad de aproximarnos al prácticamente desconocido contexto arqueológico de las pinturas rupestres ejecutadas en los abrigos de Sierra Momia, nos reveló la existencia de dos yacimientos al aire libre de un interés inesperado, que ofrecian un importante caudal de información y cuya distribución espacial se veía constreñida a áreas muy reducidas. Se trata de la Cubeta de la Paja, dentro del Conjunto rupestre del Tajo de las Figuras, y las Cuevas de Levante, lugares situados en Sierra Momia. Del conjunto global de las colecciones líticas y su deposición planteamos como hipótesis de trabajo que la funcionalidad de los yacimientos estaría acorde con los patrones de asentamientos estacionales destinados fundamentalmente al abastecimiento de recursos pétreos y su manufactura y los encuadramos cronológicamente a fases avanzadas del Solutrense Evolucionado Ibérico. 


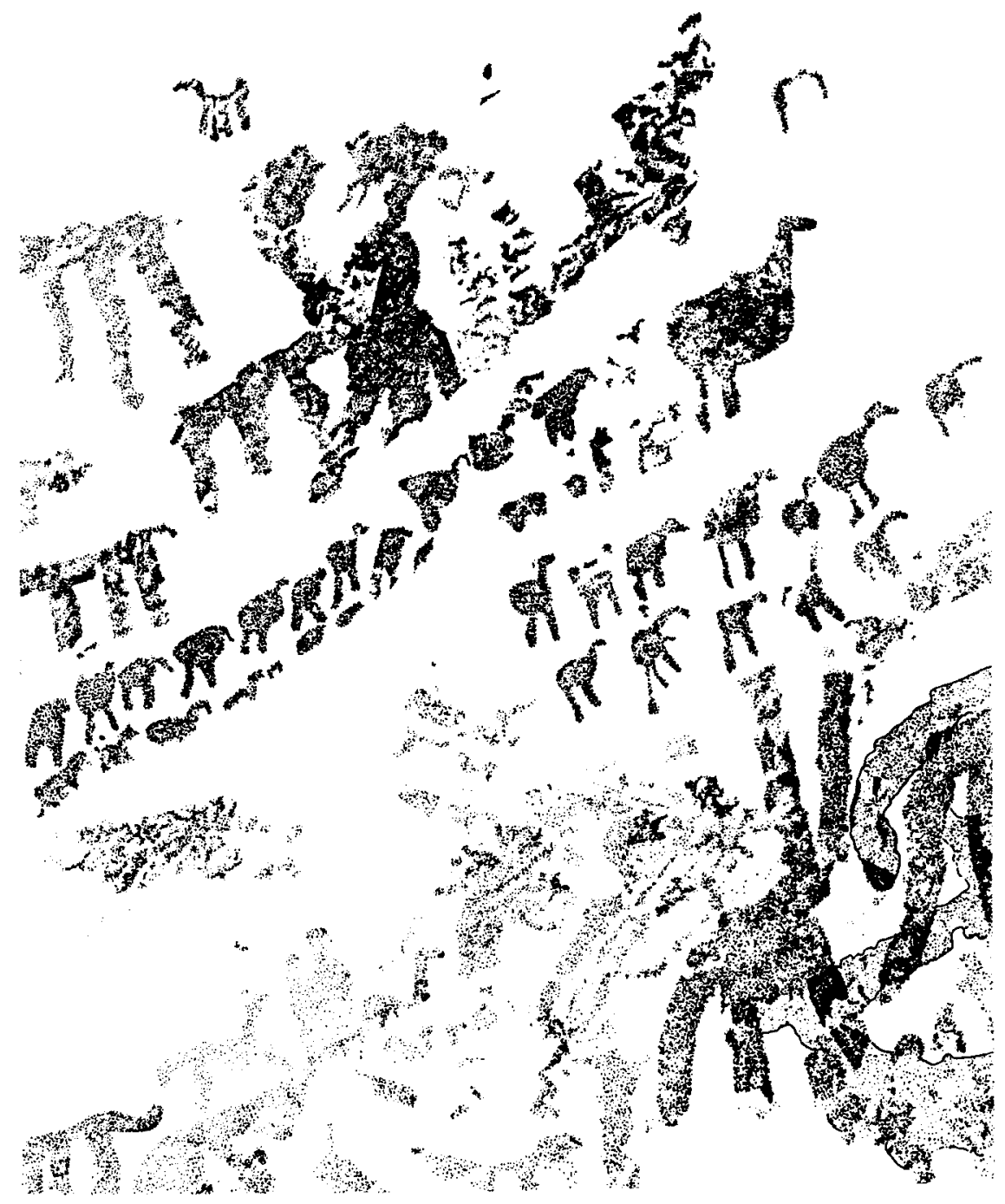

Figura 2. Detalle (reproducción) de las pinturas de la Cueva del Tajo de las Figuras. Puede apreciarse la representación de una bandada de avutardas. 
En 1991 llevamos a cabo una actuación de urgencia (Ripoll López y otros, 1991a) con el fin de diagnosticar y matizar el estado de la cuestión y plantear la intervención más idónea en función de la problemática en torno a la conservación de estos lugares. Un sondeo estratigráfico en las Cuevas de Levante y el análisis de la serie industrial confirmaron el encuadre Solutrense. Aunque la memoria definitiva de estos trabajos está todavía en fase de elaboración, podemos aportar algunos datos.

La serie industrial analizada nos muestra que el índice de raspadores alcanza un porcentaje del I.R. $=25,93 \%$, mientras que el de los buriles lo supera ampliamente (I.B. $=46,30 \%)$. En cuanto a los índices restringidos de los buriles diedros de ángulo (I.B.d. $(r)=46,30 \%$ ) y el de los buriles sobre truncatura (I.B.t. $(r)=35,19 \%)$, vemos que son relativamente similares. El índice de los útiles realizados sobre hojitas únicamente representa un $9,26 \%$ (I.h.). Sin embargo el grupo de útiles que de alguna forma puede caracterizar este conjunto industrial es el solutrense, que aunque posee un bajo porcentaje (I.G.S. $=5,56 \%$ ), junto con el grupo de los buriles y el de las hojitas, nos permite encuadrar este nivel en un horizonte cultural del Solutrense Superior Evolucionado. Como ya se ha propuesto en otras publicaciones (Ripoll López, 1988, 1989, 1991, en prensa), un encuadre tan concreto únicamente se puede efectuar cuando se posee una serie industrial suficientemente ámplia, y éste no es el caso, ya que se trata de un reducido sondeo estratigráfico provisional. Pero creemos poder adelantar que dada la existencia de una punta de aletas y pedúnculo, de una punta de muesca y de una pieza solutrense bifacial - a pesar de que todas ellas aparecen fracturadas-, que su encuadre cultural Solutrense es bastante acertado (Ripoll López y otros, 1991a). Por otra parte, la existencia de un índice tan alto de buriles, junto con las escasas hojitas, nos podría hacer pensar que se trata de un nivel Magdaleniense, pero la presencia de las piezas antes mencionadas nos ratifica en el encuadre cultural propuesto. Opinamos (Ripoll López, 1988, 1989, 1991, en prensa) que en la zona andaluza -donde la existencia del Magdaleniense Inicial y Medio no está atestiguada - estos estadios culturales se verían sustituídos por el Solutrense Superior Evolucionado.

Durante la campaña de 1991, al observar nuevamente los paneles pintados de la Cueva del Tajo de las Figuras nos dimos cuenta de la existencia de un gran número de grabados que habían pasado desapercibidos a anteriores investigaciones (Ripoll López y otros, 1991b; Mas Cornellà, 1991b; Mas Cornellà y Ripoll López, en prensa). Se trata de tres motivos figurativos - que interpretamos como posibles cérvido, équido y caprino- y otros trazos que no podemos definir formalmente. Los 
paralelos estilísticos nos llevan a situar estas representaciones en un momento Solutrense sensu lato. Éste encuadre paleolítico queda reafirmado al encontrarse infrapuestas a toda la secuencia pictográfica postpaleolítica, lo cual ratifica su antigüedad, que viene avalada también por los yacimientos próximos a los que nos hemos referido anteriormente.

Los grabados rupestres constituyen un tema complejo de definir durante los trabajos de campo. Por una parte no siempre son visibles con luz ambiental, en algunos casos en determinadas horas del día, otras sólo a partir de luces artificiales difusas o rasantes. Si a ésto unimos que la arenisca sílicea, soporte de todas las manifestaciones rupestres de la zona que nos ocupa, presenta innumerables líneas producto de su agrietamiento o erosión fácilmente confundibles con grabados, y que en muchos casos es muy difícil e incluso subjetivo definirlas o no como evidencias antrópicas, y lo imprevisible que era localizar este tipo de representaciones en los abrigos rocosos profusamente pintados del Campo de Gibraltar, puede comprenderse porqué esta técnica no fue documentada en un primer momento. Sin embargo los grabados localizados en la Cueva del Tajo de las Figuras nos obligaban a revisar todas las cavidades en las que habiamos actuado hasta ahora. Durante 1992, en septiembre, octubre y noviembre llevamos a cabo esta labor. Se observaron minuciosamente estas estaciones rupestres, intentando diferenciar la posibilidad de hallar grabados prehistóricos entre la maraña, muchas veces, de trazos recientes o grafitos, que por otra parte también documentamos. Cabe señalar que estos lugares han sido frecuentados hasta la actualidad por corcheros, cabreros, carboneros y cazadores que los han utilizado como lugar de habitación o refugio (Mas Cornellà, 1993b).

El resultado fue la localización de nuevos grabados paleolíticos en dos cavidades conocidas por sus pinturas postpaleolíticas, las Cuevas de Levante y la Cueva del Arco (Mas Cornellà, 1993b). Este último abrigo, dentro del Conjunto rupestre del Tajo de las Figuras, contiene unos trazos muy finos, largos, horizontales y curvados, uno de ellos perfectamente infrapuesto a diferentes motivos pintados. Las Cuevas de Levante constituyen un complejo de cavidades de escaso desarrollo (abrigos rocosos) alineadas en dos niveles. En una de ellas existen pinturas. Los grabados han sido encontrados en ésta y otra cavidad contigua. Observamos, en las paredes de esta última restos de figuras difícilmente definibles, unas líneas irregulares trazadas verticalmente y varias cazoletas. En el otro abrigo, muy cerca de las pinturas, hemos localizado un triángulo (Mas Cornellà y otros, 1995a).

Respecto a los monumentos megalíticos descritos por $\mathrm{H}$. Breuil y $\mathrm{W}$. Verner (1917) y C. de Mergelina (1924) en los aledaños del Conjunto rupestre del Tajo de las Figuras cabe decir que después de prospectar la 
zona (Mas Cornellà, 1991a) para confirmar o desechar la presencia de estas construcciones comprobamos que actualmente no existen. Sólo vimos afloramientos naturales de roca, sobre los cuales no podemos pensar siquiera que fueran dólmenes rupestres o semirupestres, no observando tampoco ninguna estructura intencionada antrópica ni orientación definida.

Afortunadamente no ocurre lo mismo con las estructuras divulgadas por estos mismos autores en el Aciscar-Purenque-Larraez (Sierra del Niño), cuyo estudio iniciamos también en 1991. Junto con los yacimientos de Los Charcones (Ramos Muñoz y otros, 1995a, 1995b) y Los Algarbes (Mata Almonte, 1991, 1995), a los que deben añadirse diversos lugares localizados al aire libre hace algunos años (Fernández-Llebrez Butler y otros, 1988; Ramirez Delgado y otros, 1989), constituyen los documentos que actualmente se están considerando en relación a la ocupación postpaleolítica (cultura material) por parte del hombre en las proximidades de la antigua Laguna de la Janda, aunque sería necesario intensificar las investigaciones sistemáticas en este sentido.

Nuestro proyecto, sin embargo, no ha finalizado. Entre las perspectivas futuras destacaremos las siguientes. Documentación e investigación de la totalidad de los lugares conocidos con manifestaciones rupestres prehistóricas de la zona que nos ocupa, siguiendo las directrices metodológicas que hemos definido en los informes anuales que se han venido publicando o están en prensa, desde 1986 a 1992, en el Anuario Arqueológico de Andalucia. Prospeccion sistemática del área, sierras del Campo de Gibraltar y núcleos montañosos próximos a la antigua Laguna de la Janda, con la finalidad de conocer la totalidad (o el todo probabilístico) de las estaciones con representaciones pintadas o grabadas y su relación espacial. Estamos diseñando un procedimiento a desarrollar a partir de Sistemas de Información Geográfica (Jordá Pardo y otros, 1994). Contextualización arqueológica, prospectando sistemáticamente la zona y realizando determinados sondeos estratigráficos en cavidades o yacimientos al aire libre en donde hemos apreciado la existencia de relleno y/o materiales arqueológicos en superficie. Estudio y análisis de los pigmentos y las técnicas de ejecución de los motivos, dataciones directas, aplicación de procedimientos fotográficos basados en radiaciones de longitud de onda fuera del espectro visible sobre determinadas figuras ..., cuestiones que hemos comenzado a tener en cuenta, junto a la problemática de las estratigrafías cromáticas, las relaciones entre temática y estilo, las tipologías ..., y que observadas de manera global pueden aportarnos nuevos datos a la interpretación de estos documentos. 


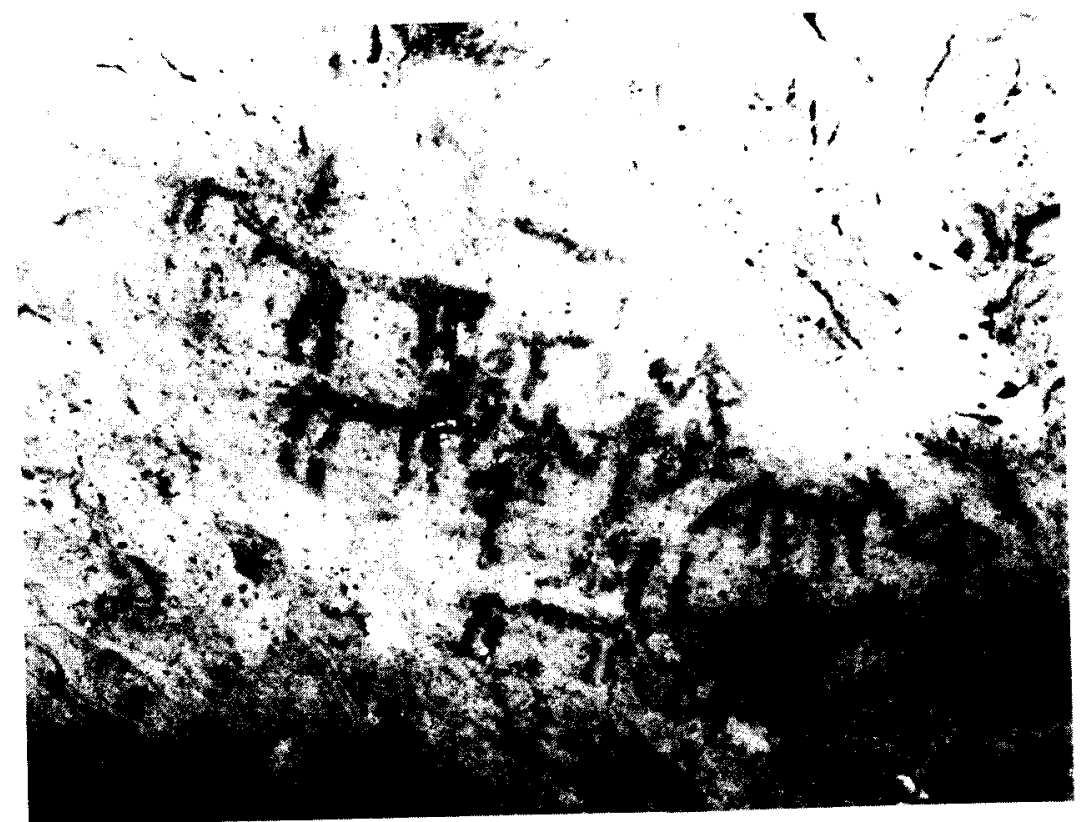

Figura 3. Fragmento del panel principal del Gran Abrigo de Bacinete.

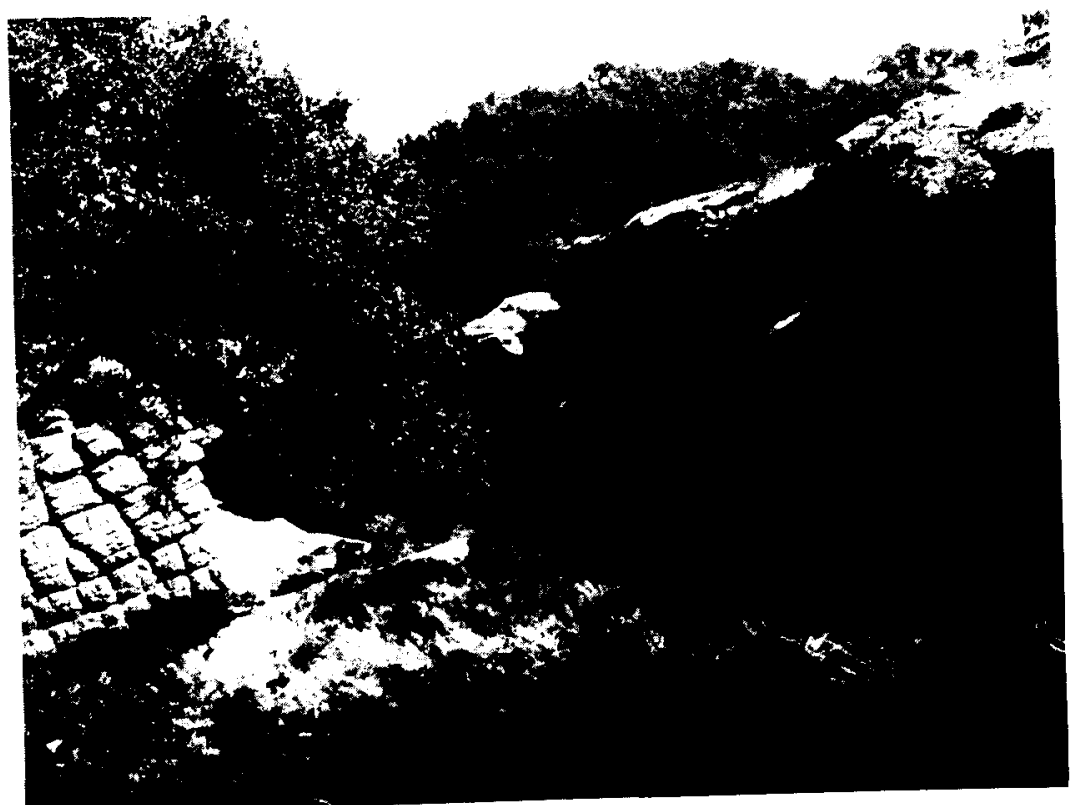

Figura 4. Vista general de los Abrigos de Bacinete. 


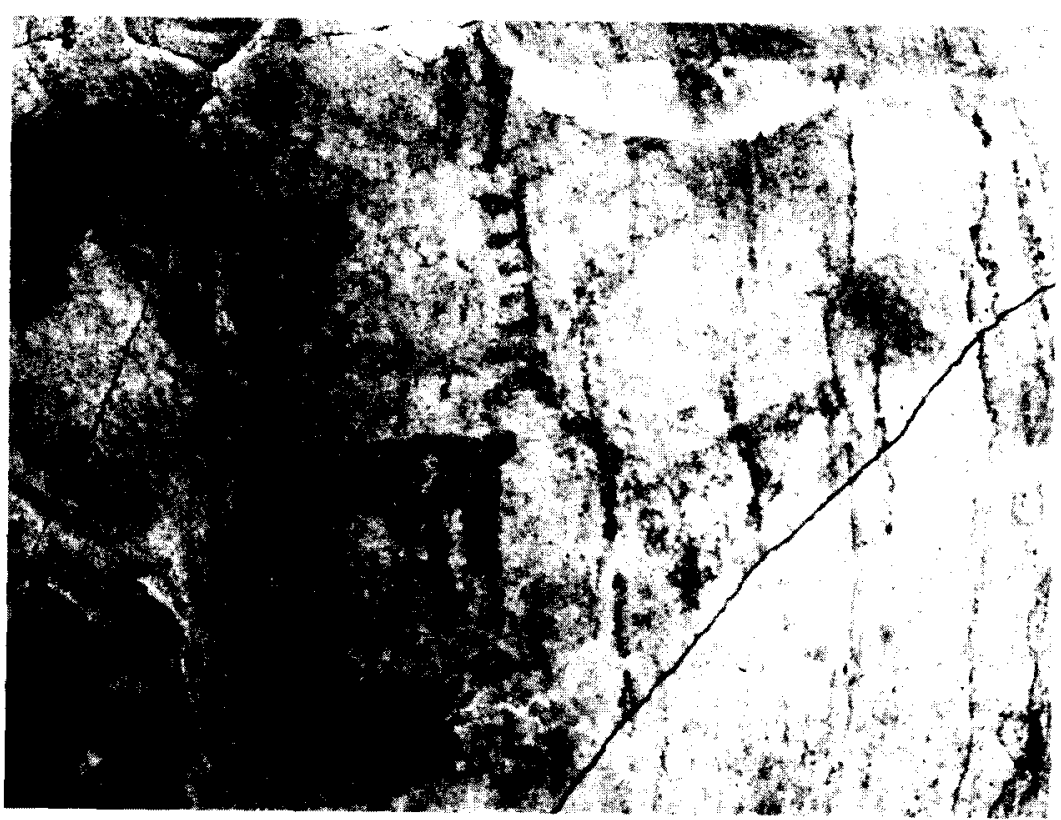

Figura 5. Ciervos pintados en la Cueva de Palomas 1.

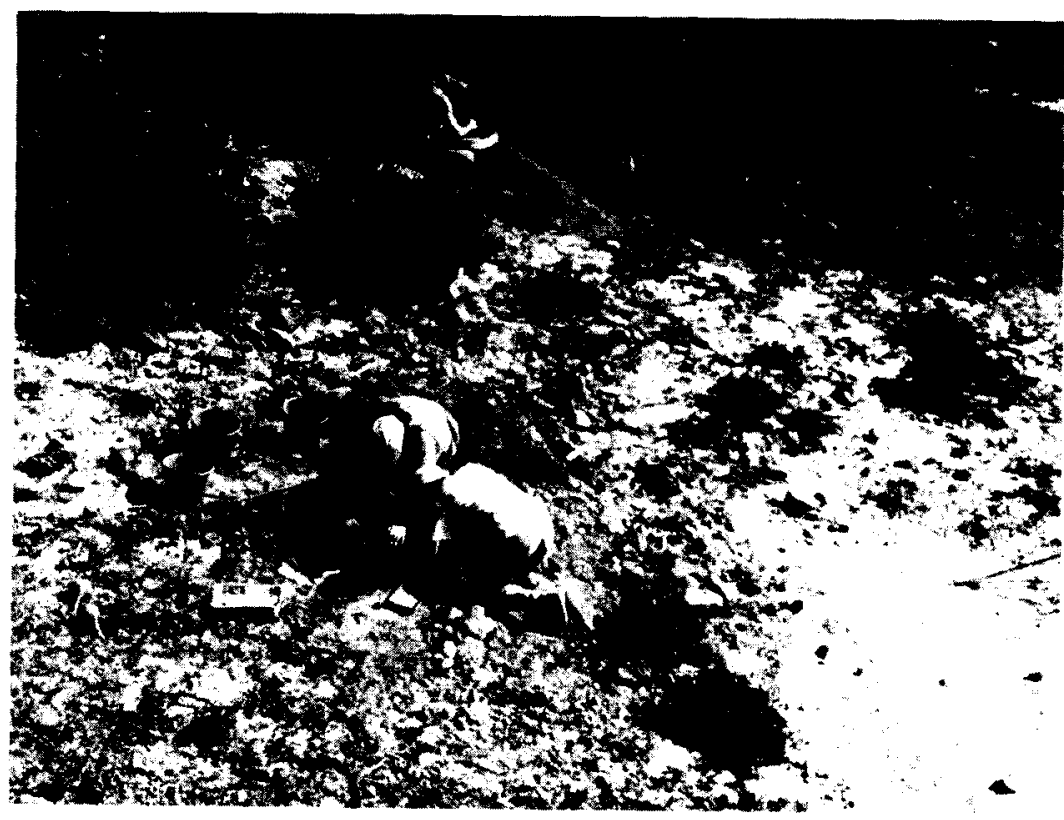

Figura 6. Trabajo de excavación en Cuevas del Levante. 
Entre los resultados obtenidos por el proyecto de investigación arqueológica Las manifestaciones rupestres prehistóricas de la zona gaditana cabe señalar que se ha constatado una clara ocupación por parte del hombre de esta zona durante el Paleolítico Superior. Estos descubrimientos e hipótesis de trabajo han venido a aumentarse y han sido confirmadas por el reciente descubrimiento de la Cueva del Moro (Mas Cornellà y otros, 1995a, 1996; Ripoll López y Mas Cornellà, 1996), aunque también debe destacarse que en este sentido estamos analizando otros lugares como las Cuevas de Atlanterra y de los Ladrones o Pretinas 1 y 4 , las cuales ofrecen una cierta complejidad técnica y temática que hay que considerar con precaución.

Por lo que respecta al arte postpaleolítico creemos que la documentación que estamos elaborando permitirá dar a conocer próximamente un auténtico corpus sobre las representaciones pintadas y grabadas en los abrigos rocosos del Campo de Gibraltar. Esta nueva interpretación aporta también nuevas hipótesis de trabajo, basadas en estudios técnicos - pigmentos, aglutinantes, procesos de aplicación, codificación de las gamas cromáticas ...- y estilísticos -considerando las superposiciones, infraposiciones y yuxtaposiciones, la temática, los paralelos muebles y cerámicos... - que plantean nuevas conclusiones con diferentes connotaciones culturales y cronológicas (Mas Cornellà, en prensa).

\section{BIBLIOGRAFÍA}

Acosta, P::

- 1968, La pintura rupestre esquemática en España, Facultad de Filosofía y Letras de la Universidad de Salamanca (Memorias del Seminario de Prehistoria y Arqueología, 1), Salamanca, $250 \mathrm{p}$.

- 1984, "El arte rupestre esquemático ibérico: problemas de cronologia preliminares", (J. Fortea, editor): Scripta Praehistorica Francisco Jordá Oblata, Ediciones Universidad de Salamanca (Acta Salmanticensia lussu Senatus Vniversitatis Edita, Filosofía y Letras, 156), Salamanca, p. 31-61.

ALmagro Gorbea, M.:

- 1988, «Representaciones de barcos en el arte rupestre de la Península lbérica. Aportación a la navegación precolonial desde el Mediterráneo Oriental', (E. Ripoll Perelló, editor): Actas del Congreso Internacional El Estrecho de Gibraltar. Ceuta, 1987. I: Prehistoria o Historia de la Antiguedad, Universidad Nacional de Educación a Distancia, Madrid, p. 389-398.

BALDELLOU, $V$.

- 1989, "/l Reunión de Prehistoria aragonesa: La terminología en el arte rupestre post-paleolítico", Bolskan, 6, p. 5-14.

Barroso Ruiz, C.

- 1980, "Nuevas pinturas rupestres en Jimena de la Frontera (Cádiz): Abrigo de Laja Alta", Zephyrus, $X X X-X X X I, p .23-42$.

Breult, $H$.

- 1921, "Nouvelles cavernes ornées paléolithiques dans la province de Malaga", L'Anthropologie, XXXI, p. 239-253.

- 1922, "Palaeolithic man at Gibraltar: new and old facts". The Journal of the Royal Anthropological Institute of Great Britain and Ireland, LII, p. 46-54. 
- 1974, Quatre cents siecles d'art pariétal. Les cavernes ornées de l'age du renne, Editions Max Fourny Art et Industrie, Paris (segunda edición), $413 \mathrm{p}$.

BREUIL, H. Y BURKITT, M. C.

- 1929, Rock paintings of Southern Andalusia. A description of a Neolithic and Copper Age art group, Clarendon Press, Oxford, XII + $88 \mathrm{p}$.

BREUIL, $H$. Y VERNER, W.

- 1917, "Découverte de deux centres dolméniques sur les bords de la Laguna de la Janda (Cadix)", Bulletin Hispanique, XIX, p. 157-188.

Cabré, J. Y Hernández-Pacheco, $E$.

- 1914, "Avance al estudio de las pinturas prehistóricas del extremo Sur de España (Laguna de la Janda)", Trabajos de la Comisión de Investigaciones Paleontológicas y Prehistóricas, 3, 35 p.

Corzo Sánchez, R. y Giles Pacheco, F.

- 1978, «El Abrigo de la Laja Alta», Boletín del Museo de Cádiz, I, p. 19-35.

Fernández-Llebrez Butler, C., Mateos Alonso, V. y Ramirez Delgado, J.

- 1988, "Los yacimientos paleolíticos de la Depresión de la Janda (provincia de Cádiz)", (E. Ripoll Perelló, editor): Actas del Congreso Internacional El Estrecho de Gibraltar. Ceuta, 1987. I: Prehistoria e Historia de la Antiguedad, Universidad Nacional de Educación a Distancia, Madrid, p. 87-96.

FONTBOTÉ, J. M.

- 1983, "La Cordillera Bética. Zonas internas y unidades adyacentes", Libro Jubilar J. M. Rlos. Geología de España, Instituto Geológico Minero de España, Madrid, p. 251-343.

FONTBOTÉ, J. M. Y VERA, J. A.

- 1983, "La Cordillera Bética. Introducción", Libro Jubilar J. M. Rios. Geología de España, Instituto Geológico Minero de España, Madrid, p. 205-218.

ForTea PÉREZ, F. J.

- 1978, "Arte paleolítico del Mediterráneo español», Trabajos de Prehistoria, 35, p. 99-149.

GaVALA, J.

- 1916, “Regiones petrolíferas de Andalucia», Boletín del Instituto Geológico Minero de España, 37, p. 33-208.

- 1929, "La Geología del Estrecho de Gibraltar», Boletín del Instituto Geológico Minero de España, 51, p. 3-36.

HeRnández-PACHECo, E. y CABRÉ, J.

- 1913, "La depresión del Barbate y sus estaciones prehistóricas", Boletín de la Real Sociedad Española de Historia Natural, XIII, p. 349-359.

ITGE

- 1991, Mapa Geológico de España, Escala 1:50.000, Tahivilla, 1.074 (13-47), Instituto Tecnológico Geominero de España, Madrid.

Jordá Pardo, J. F., Lain Huerta L. y Mas Cornella, M.

- 1994, "Sistemas de Información Geográfica, Geoarqueología y Prospección Arqueológica. Una propuesta metodológica para la localización exhaustiva de yacimientos con arte rupestre en un sector de la provincia de Cádiz", (J. F. Jordá Pardo, editor): Geoarqueologl'a (Actas de la 2." Reunión Nacional de Geoarqueología. ITGE, Madrid, 14, 15 y 16 de diciembre de 1992), Instituto Tecnológico Geominero de España-Asociación Española para el Estudio del Cuaternario, Madrid, p. 439-448.

Mas CORNELLÀ, M.

- 1986, "Informe sobre el estudio del Conjunto rupestre del Tajo de las Figuras (Medina Sidonia, Cádiz)», Anuario Arqueológico de Andalucia, 1986. II: Actividades Sistemáticas. Informes y Memorias, $p$. 258-260.

- 1986-1987, "Los grabados de la Cueva del Arco (Conjunto rupestre del Tajo de las Figuras) y del Abrigo del Tajo de Albarianes (Medina Sidonia Cádiz)", Ars Praehistorica, V-VI, p. 247-252.

- 1987, “Informe sobre el estudio del Conjunto rupestre del Tajo de las Figuras (Medina Sidonia, Cádiz), 1987", Anuario Arqueológico de Andalucia, 1986. Il: Actividades Sistemáticas. Informes y Memorias, p. 398-400.

- 1988a, «El Conjunto rupestre del Tajo de las Figuras. Estado actual de las investigaciones en Sierra Momia (Cádiz)", (E. Ripoll Perelló, editor): Actas del Congreso Internacional EI Estrecho de Gibraltar. Ceuta, 1987. I: Prehistoria e Historia de la Antiguedad, Universidad Nacional de Educación a Distancia, Madrid, p. 293-303. 
- 1988b, “Las manifestaciones rupestres prehistóricas de la zona gaditana. 1988: Sierra Momia", Anuario Arqueológico de Andalucia, 1988. Actividades Sistemáticas. Informes y Memorias, p. 213-220.

- 1988-1989: "Las manifestaciones rupestres postpaleolíticas de Sierra Momia (Cádiz). Algunos apuntes", Ars Praehistica, VII-VIII (= Homenaje al Profesor Eduardo Ripoll Perelló), p. 195-199.

- 1989a, "Algunas consideraciones sobre la conservación del arte prehistórico en el Conjunto rupestre del Tajo de las Figuras (Cádiz)", (A. Beltrán Martínez, editor): XIX Congreso Nacional de Arqueología. Castellón de la Plana, 1987. II: Arte rupestre y Valle del Ebro, Secretaría General de los Congresos Arqueológicos Nacionales, Zaragoza, p. 161-167.

- 1989b, "Las manifestaciones rupestres prehistóricas de la zona gaditana. 1989: Sierra Momia y Valle del Río de las Cañas o Palmones. Informe" Anuario Arqueológico de Andalucía, 1989. Actividades Sistemáticas. Informes y Memorias, p. 353-359.

- 1990: «Proyecto de investigación arqueológica Las manifestaciones rupestres prehistóricas de la zona gaditana. 1990: Reproducción y estudio directo del arte rupestre en Sierra Momia y Valle del Rio de las Cañas o Paimones", Anuario Arqueológico de Andalucía, 1990. Acfividades Sistemáticas. Informes y Memorias, p. 352-358.

- 1991a, "Prospecciones arqueológicas en Sierra Momia (Cádiz)", Anuario Arqueológico de Andalucia, 1991. Il: Actividades Sistemáticas. Informes y Memorias, p. 93-98.

- 1991b, "Documentación e investigación de las manifestaciones artísticas en las Cuevas de Palomas, Abrigos de Bacinete y Conjunto rupestre del Tajo de las Figuras (Cádiz)", Anuario Arqueológico de Andalucía, 1991. Il: Actividades Sistemáticas. Informes y Memorias, p. 99-104.

- 1993a, "El Abrigo de la Laja Alta y el arte prehistórico del Campo de Gibraltar", I Jornadas Seminario Permanente de Historia y Arqueología, Ayuntamiento de Jimena de la Frontera, Jimena de la Frontera, p. 9-14.

- 1993b, «Proyecto: Las manifestaciones rupestres prehistóricas en la zona gaditana. El arte prehistórico en las sierras del Campo de Gibraltarm, (J. M. Campos CARRAsco y F. Nocete CALvo, editores): Investigaciones arqueológicas en Andalucia, 1985-1992. Proyectos, Dirección General de Bienes Culturales de la Consejerla de Cultura y Medio Ambiente de la Junta de Andalucia, Huelva, p. 263-271.

- en prensa, "Naturalism and Schematism in the post-palaeolithic rock art of the Southern Iberian Peninsula", (R. G. Bednarik y F. D'Errico, editores): News 95 - International Rock Art Congress. Torino, August September 1995. Symposium 1A: New approaches, Centro Studi e Museo di Arte Preistorica, Torino.

- inédito, Catálogo de yacimientos con pinturas rupestres en Andalucía, Dirección General de Bienes Culturales de la Consejería de Cultura de la Junta de Andalucía, Sevilla, 1995, 2.207 p. (8 volúmenes).

MAS CORNELLȦ, M. Y RIPOLL LóPEZ, S.

- en prensa, "Los grabados rupestres de Cádiz", Actes del ler Congrés Internacional de Gravats Rupestres y Murals (Homenatge a Liuis Diez-Coronel). Lleida, 1992, Institut d'Estudis llerdencs, Lleida.

MAS CORNELLA, M. Y SANCHIDRIÁN TORTI, J. L.

- 1990, «Proyecto de investigación arqueológica Las manifestaciones rupestres prehistóricas de la zona gaditana. 1990: Prospección arqueológica superficial en las Cuevas de Levante y el Conjunto rupestre del Tajo de las Figuras (Sierra Momia)", Anuario Arqueológico de Andalucia, 1990. II: Actividades Sistemáticas. Informes y Memorias, p. 359-370.

mas Cornella, M. y Torra Colell, G.

- 1990, "Arte rupestre en Cádiz. Documentación e investigación", Revista de Arqueología, 113, p. 14-22.

- 1995, "Avance al estudio de las manifestaciones rupestres postpaleolíticas de Cádiz: estado actual de las investigaciones", (E. Ripoll Perello y M. Ladero Quesada, editores): Actas del II Congreso Internacional El Estrecho de Gibraltar. Ceuta, 1990. I: Crónica y Prehistoria, Universidad Nacional de Educación a Distancia, Madrid, p. 181-192.

mas Cornella, M., Jordá Pardo, J. F., Cambra Sánchez, J., Mas Riera, J. y lombarte Caraera, A.

- 1994, "La conservación del arte rupestre en las sierras del Campo de Gibraltar. Un primer diagnóstico", Espacio, Tiempo y Forma. Serie I: Prehistoria y Arqueología, 7, p. 93-128. 
Mas Cornelà, M., Ripoll lópez, S., Bergmann, L., Paniagua Pérez, J. P., López Moreno de REDROJO, J. R. Y MARTOS ROMERO, J. A.

- 1996, "La Cueva del Moro. El arte paleolítico más meridional de Europa", Revista de Arqueología, 177, p. 14-21.

mas Cornella, M., Ripoll lópez, S., martos Romero, J. A., Paniagua Pérez, J. P., lópez MORENo de REDROJO, J. R. Y BergmanN, L.

- 1995a, "Estudio preliminar de los grabados rupestres de la Cueva del Moro (Tarifa, Cádiz) y el arte paleolítico del Campo de Gibraltar", Trabajos de Prehistoria, 52/2, p. 61-81.

mas Cornellà, M., Torra Colell, G., Ripoll lópez, S., Gavilan Ceballos, B., Vera Rodriguez, J. C. Y JORDA PARDO, J. F.

- 1995b, «El poblamiento prehistórico en las sierras próximas a la antigua Laguna de la Janda" (J. M. Recio Espejo, J. C. Castro Román y A. Santiago Pérez, editores): jornadas de Campo en la Depresión de la Janda (Cádiz). 19, 20 y 21 de mayo de 1995, Asociación Española para el Estudio del Cuaternario - AEQUA-GAC, Córdoba, p. 92-104.

MATA ALMONTE, E.

- 1991, "Informe sobre la intervención arqueológica en el yacimiento de Los Algarbes, Tarifa (Cádiz). Campaña 1990", Anuario Arqueológico de Andalucía, 1991. III: Actividades de Urgencia. Informes y Memorias, p. 83-93.

- 1995, "Informe sobre la intervención arqueológica en la necrópolis prehistórica de Los Algarbes (Tarifa, Cádiz). Campaña 1990"; (J. M. Recio Espejo, J. C. Castro Román y A. Santiago Pérez, editores): Jornadas de Campo en la Depresión de la Janda (Cádiz). 19, 20 y 21 de mayo de 1995, Asociación Española para el Estudio del Cuaternario - AEQUAGAC, Córdoba, p. 105-126.

MERGELINA, C. DE

- 1924, "Los focos dolménicos de la Laguna de la Janda", Memorias de la Sociedad Española de Antropología, Etnografía y Prehistoria, III, p. 97-126.

MOLINA, $V$.

- 1913, "Arqueología y prehistoria de la provincia de Cádiz en Lebrija y Medinasidonia", Boletín de la Real Academia de la Historia, LXII, p. 554-562.

Pérez Ramos, L.

- 1995, “Avance sobre un hallazgo prehistórico en las terrazas del Río Palmones", Almoraima, 13 (= III Jornadas de Historia del Campo de Gibraltar), p. 65-70.

POSAC MON, C.

- 1975, "Los Algarbes (Tarifa). Una necrópolis de la Edad del Bronce", Noticiario Arqueológico Hispánico, 4, p. 87-119.

Ramos Muñoz, J., Castañeda Fernández, V., Pérez Rodríguez, M., Lazarich González, M., Martínez Peces, C., Montañés Caballero, M., lozano Moya, J. M. y Caloerón Estrada, D.

- 1995a, "Los Charcones. Un poblado agrícola del III y II milenios a. de C. Su vinculación con el foco dolménico de la Laguna de la Janda", Almoraima, 13 (= III Jornadas de Historia del Campo de Gibraltar), p. 33-50.

- 1995b, "Los Charcones. Un poblado agrícola del III y II milenios a. de C. Su vinculación con el foco dolménico de la Laguna de la Janda", (J. M. Recio Espejo, J. C. Castro Román y A. Santiago Pérez, editores): Jornadas de Campo en la Depresión de la Janda (Cádiz). 19, 20 y 21 de mayo de 1995 Asociación Española para el Estudio del Cuaternario - AEQUA-GAC, Córdoba, p. 70-91.

Ramos muñoz, J., Castañeda fernández, V., Pérez Rodríguez, M., Lazarich González, M., Montañés Caballero, M., Arroquia Rodriguez, M. !., Blanes Delgado, C., Martínez Peces, C., Domínguez Bella, S., Gracia Prieto, J., Morata Céspedes, D., Pérez Ramos, L., Gómez arroquia, M. I., Herrero lapaz, N., Reina Martínez, A., Cantalejo duarte, P., Brito Guerrero, M. M., Guzmán Fernández, J. C., Calderón Estrada, D., lozano Moya, J. M. Y SoRIANO GaLIANA, M.

- 1995c, El Paleolitico Superior Final del Río Palmones (Algeciras Cádiz). Un ejemplo de la tecnología de las comunidades especializadas de cazadores-recolectores, Instituto de Estudios Campogibraltareños Algeciras, $251 \mathrm{p}$.

Ramírez Delgado, J. R., Fernández-Llebrez Butler, C. y Mateos Alonso, V.

- 1989, «Aproximación al estudio del Cuaternario de la Laguna de la Janda (Cádiz)», (F. Diaz del Olmo y J. Rodríguez Vidal, editores): El Cuaternario en Andalucía Occidental, Asociación Española para el Estudio del Cuaternario (AEQUA Monografías, 1), Sevilla, p. 105-111. 
RiPoll López, S.

- 1988, "La Cueva de Ambrosio (Vélez-Blanco Almería) y su posición cronoestratígráfica en el Mediterráneo Occidental», British Archaeological Report, 462 (2 vols.), Oxford, 596 p.

- 1989, "Le gisement de La Cueva de Ambrosio: Nouveaux apports au Solutréen de la Péninsule lbériquem, L'Anthropologie 92, p. 851-886.

- 1991, "Le solutréen dans le Sud de la Péninsuie Ibérique", Colloque International sur les industries a pointes foliacées du Paléolithique superieur europeen, organizado por el Instytut Archeologii Universytet Jagiellonski, en Cracovia (Polonia), 25 de Septiembre a 10 de Octubre de 1989, Les feuilles de pierre, E.R.A.U.L., 42, Liege p. 449-465.

- en prensa, "Die stratigraphisch-zeitliche Einordnung der Cueva de Ambrosio in Almeria, (Sudspanieü)", Congreso Internacional de la Unión Internacional de Ciencias Prehistóricas y Protohistóricas (U.I.S.P.P.), Mainz 1987.

RIPOLL LÓPEZ, 5. Y MAS CORNELLȦ, M.

- 1996, "Art paléolithique dans l'extrême Sud de l'Europe - Paleolithic art in the far South of Europe", International Newsletter on Rock Art, 13, p. 7-10.

Ripoll López, 5., Mas Cornellà, M. Y Perdigones Moreno, L.

- 1991a, "Actuaciones de urgencia en las Cuevas de Levante y Cubeta de la Paja (Sierra Momia, Benalup, Cádiz)", Anuario Arqueológico de Andalucía, 1991. III: Actividades Sistemáticas. Informes y Memorias, p. 105-110.

Ripoll lópez, 5., Mas Cornellà, M. y Torra Colell, G.

- 1991b, "Grabados paleolíticos en la Cueva del Tajo de las Figuras (Benalup)", Espacio, Tiempo y Forma. Serie I: Prehistoria y Arqueología, 4, p. 111-126.

Ripoll Perelló, E.

- 1990, "Acerca de algunos problemas del arte rupestre postpaleolítico en la Península Ibérica", Espacio, Tiempo y Forma. Serie l: Prehistoria y Arqueología, 3, p. 71-104.

SANCHIDRIAN TORTI, J. L. Y MAS CORNELLA, M.

- 1995, “Discusiones en torno al considerado arte paleolítico del Campo de Gibraltar (Cádiz)", (E. Ripoll Perelló y M. Ladero Quesada, editores): Actas del II Congreso Internacional EI Estrecho de Gibraltar. Ceuta, 1990. I: Crónica y Prehistoria, Universidad Nacional de Educación a Distancia, Madrid, p. 73-86.

SANTIAGo VILCHEZ, J. M.

- 1979-1980, "La Cueva de las Palomas en el arte paleolítico del Sur de España", Boletín del Museo de Cádiz, II, p. 5-11.

VERNER, $W$.

- 1914a, "Prehistoric man in Southern Spain. I", Country Life, XXXV/911, p. 901-904.

- 1914b, "Prehistoric man in Southern Spain. Il», Country Life, XXXVI/914, p. 41-45.

- 1914c, "Prehistoric man in Southern Spain. III', Country Life, XXXVI/916, p. 114-118

WAECHTER, J.

- 1953, "The excavation of Gorham's Cave and its relation to the prehistory of Southern Spain", Archivo de Prehistoria Levantina, IV (= Homenaje a D. Isidro Ballester Tormo), p. 21-24.

- 1964, "The excavation of Gorham's Cave, Gibraltar, 1951-54", Bulletin of the Institute of Archaeology, 4, p. 189-221. 